\title{
The EU DEMO equatorial outboard limiter - design and port integration concept
}

\author{
Thomas Franke ${ }^{\mathrm{a}, \mathrm{b}}$, Christian Bachmann ${ }^{\mathrm{a}}$, Wolfgang Biel ${ }^{\mathrm{c}}$, Fabio Cismondi ${ }^{\mathrm{a}}$, Oliver Crofts ${ }^{\mathrm{d}}$, Aljaz \\ Cufar $^{\mathrm{e}}$, Gianfranco Federici ${ }^{a}$, Winder Gonzalez ${ }^{\mathrm{c}}$, Richard Gowland ${ }^{\mathrm{d}}$, Gregory Keech ${ }^{\mathrm{a}}$, Rocco \\ Mozzillof, Francesco Maviglia a, Massimo Roccella ${ }^{\mathrm{g}}$, Mikhael Tokar ${ }^{\mathrm{c}}$, Zsolt Vizvary ${ }^{\mathrm{d}}$ \\ ${ }^{a}$ EUROfusion Power Plant Physics and technology (PPPT) department, Garching, Germany \\ ${ }^{b}$ Max-Planck-Institut für Plasmaphysik, Garching, Germany \\ 'Institute of Energy and Climate Research, Forschungszentrum Jülich GmbH, Jülich, Germany \\ ${ }^{d}$ Culham Centre for Fusion Energy, Abingdon, OX14 3DB, Oxfordshire, U.K. \\ ${ }^{e}$ Reactor Physics Department, Jožef Stefan Institute, Jamova cesta 39, SI-1000, Ljubljana, Slovenia \\ ${ }^{f}$ CREATE, Engineering School of Basilicata University, Campus Macchia Romana, 85100, Potenza, Italy \\ ${ }^{g}$ LTCalcoli Srl, 23807 Merate (LC), Italy
}

\begin{abstract}
The equatorial outboard limiters (also called outboard midplane limiters (OMLs)) are an essential part of the DEMO wall protection concept. Limiters are foreseen in different areas of the DEMO first wall, namely in the equatorial ports, on the high-field side, in vertical ports and additional protection limiters between equatorial and lower ports. The limiters shall prevent the plasma to touch the first wall of the breeding blankets during all plasma transients.

The port integration concept of the OMLs, used for plasma ramp-up/-down, is explained including i) thermal, structural and electromagnetic loads, ii) neutronic requirements and related material properties, iii) remote handling considerations, iv) space and mass constraints and v) the required alignment precision to allow equal distribution of the heat exposure amongst the individual of the plasma facing (PFC) limiter components.

While the hot fusion plasma during ramp-up is impinging directly on the limiter, its PFC components temperature is rising and can be measured by means of either thermocouples or by infrared (IR) thermography an estimation of the heat flux on the contact point can be made. This is the basis for the proposed alignment strategy.
\end{abstract}

Keywords: DEMO, equatorial limiter, infrared thermography, wall protection, ramp-up/-down limiter

\section{Introduction}

The protection of the first wall (FW) of DEMO, a first of a kind DEMOnstration fusion power plant [1], is one of the key design integration issues [2], [3] in the scope of the Power Plant Physics and Technology (PPPT) department of the EUROfusion Consortium. In order to protect the breeding blankets (BBs) FW from charged particle loads, protection limiters are foreseen [4]. Fig. 1 shows the equatorial outboard limiter (also called outboard midplane limiter (OML)) and the upper limiter (UL) in the upper ports. Additional limiters on high field side and below the OML are presently under study. According to [4], and due to the present 16 toroidal field (TF) coils considered for the DEMO baseline, at present a total of 4 OMLs are being considered, arranged toroidally symmetric around the DEMO tokamak. The OMLs shall mainly serve for the ramp-up and -down of the plasma current during the initial and final limiter configuration, respectively. Further studies shall clarify if a fewer number of OMLs is still feasible related to power handling. The main requirements are described below (chapter 2). The paper presents the OML design and its integration into the vacuum vessel (VV) with the $\mathrm{BB}$, including the cooling layout, the radial alignment concept, the neutron shielding (chapter 3), the remote maintenance (RM) strategy (chapter 4) and its diagnostic system and alignment strategy (chapter 5). The development work of these limiters is on-going and further optimizations and design improvements are under discussion. The work presented here is a result of a 12 months interdisciplinary effort.

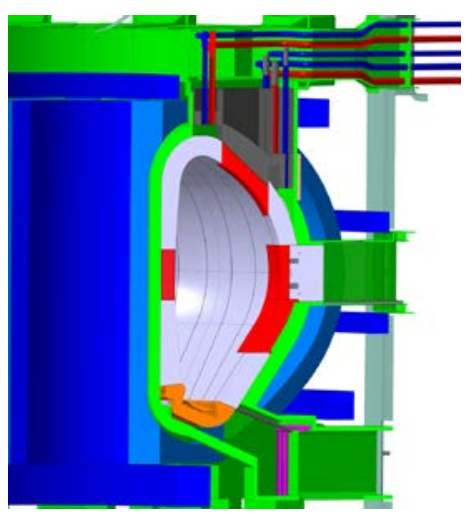

Fig. 1. DEMO poloidal cross-section with inboard-midplane, upper, outboard midplane, and outboard lower limiters shown in red, breeding blanket (grey), divertor (sandy brown), VV and ports (green), TF coils (light blue), central solenoid transformer and poloidal field coils (dark blue), pipes (red, blue and magenta). [3] (For interpretation of the references to color in this figure legend, the reader is referred to the web version of this

\section{Main requirements} article).

The requirements for the OML can be divided in several categories, see Table 1.

Table 1. Requirements for the limiter design. 
High heat flux components for plasma ramp-up

Tungsten/copper-based water cooled plasma-facing components (PFCs) are described in [5].

Remote maintenance (RM) requirements for limiter port plug and PFCs

- Minimize the time required for port plug assembly, removal and repair.

- Minimize the time required for replacement of plasma-facing components.

- Minimize size and weight of port plug.

- Minimize components in port cell and port interspace, which are to be removed prior to port plug maintenance.

- Minimize penetrations of port closure plate.

Radial protrusion to BB FW and adjustability

- Protrusion $\sim 20 \mathrm{~mm}$ to BB FW.

- Adjustment range: $\pm 5 \mathrm{~mm}$ (sum of asymmetric ferromagnetic forces $\pm 2 \mathrm{~mm}$ and relative radial alignment precision with other ramp-up limiters: $\pm 3 \mathrm{~mm}$ due to expected manufacturing assembly tolerances and thermal expansion).

- Adjustment precision $\sim 0.5 \mathrm{~mm}$, assumed to be $1 / 10$ of the typical e-folding length of the scrape of layer, for outboard limited plasmas in DEMO expected to be $\sim 6 \mathrm{~mm}$ [6]

Neutron and radiation shielding

- Irradiation damage limits of limiter and VV, nuclear heating limits of superconducting coils, shut-down dose rate (SDDR) limits, see chapter 3.5 .

\section{Design concept}

\subsection{Port integration}

The poloidal extension of the limiter is based on physics studies and ensures the plasma to remain in contact with the limiter PFCs until the plasma x-point is formed [4]. An example for a limiter configuration during plasma ramp-up is shown in Fig. 2.

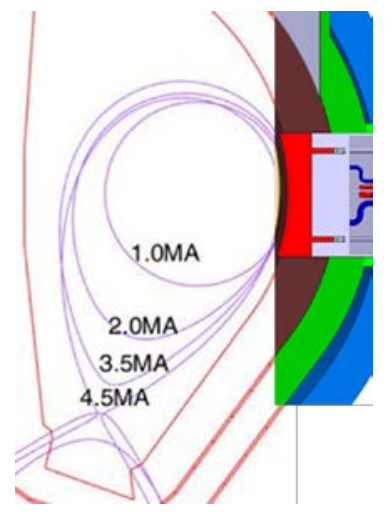

Fig. 2. Limiter configuration during plasma ramp-up. The poloidal extension of the OML prevents plasma - BB contact during ramp-up (plasma boundaries at different ramped plasma currents are indicated, [7]). The alignment of the actuators is required to enable precise relative position between 4 of such toroidally distributed (around the outer midplane) limiters.

The integration into the $\mathrm{BB}$ requires a shape which is adapted to the complex internal structure of the BB box design. Therefore the VV port and port plug are of rectangular shape, which consequently leads to rectangular cryostat and bioshield bellows. A slim toroidal cut-out in the $\mathrm{BB}$ is required to maintain the vertical integrity of the $\mathrm{BB}$ segments (the same as used for electron cyclotron (EC) BB integration [8]), which also requires an offset of the limiter port plug from the sector center-line, see Fig. 3. As the configuration is consequently not fully radial, it needs a PFC front part with a small toroidal inclination. Other than in case of the ITER port plugs the port plug mechanical flange is not at the same location as the vacuum closure plate. This allows the cooling pipes to penetrate the permanent part of the port structure next to the closure plate rather than penetrating the closure plate itself, see Fig. 3. The pipes run outside of the access space necessary to install or remove the limiter port plug and are then connected to vertical pipe manifolds. The vertical pipe manifolds connect the cooling circuits of the upper port limiter with the OML and the divertor and they are placed between cryostat and bioshield, see Fig. 3 right side.

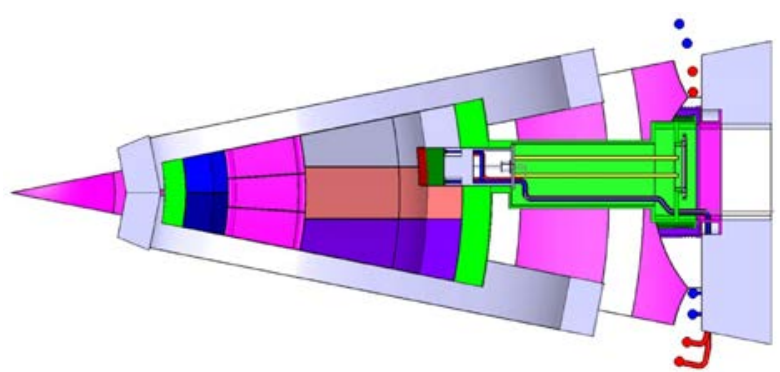

Fig. 3. Section view of DEMO (toroidal cut) with OML. The pipes (blue, red) run aside at the port and penetrate the primary vacuum next to the port closure plate in a permanent installation. In the figure, right side, also can be seen the pipe manifolds running vertically between the cryostat (pink) and the bioshield (grey). (For interpretation of the references to color in this figure legend, the reader is referred to the web version of this article).

Fig. 4 shows the overall arrangement of the OML. The PFCs are mounted on a shield block made out of Eurofer (to ensure reduced material activation and to resist the high neutron irradiation). The port plug is short to have a small weight for easier RM. The port plug is protected from neutrons by the shield block and can therefore be made out of less expensive stainless steel. The port plug plate and the port closure plate are separated in functions by design. The port closure plate is the vacuum boundary that has no other mechanical functions and is also free from any penetrations to simplify the opening of the port.

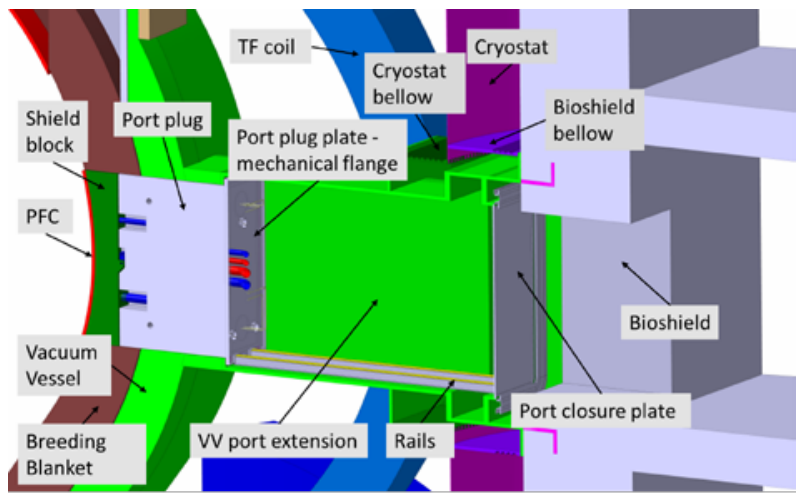

Fig. 4. Overall arrangement of the OML.

\subsection{Cooling concept}

The cooling concept of the limiter provides two different cooling circuits, one for the CuCrZr-based PFCs and another one for the Eurofer-made shield block and the port plug of stainless steel. This is equivalent to the choice made in the divertor [9], [10]. For details on inlet/outlet temperatures and pressures, see Fig. 5. The cooling circuits of the UL, the OML and the divertor operate in DEMO at the same conditions, thus it 
simplifies the overall DEMO plant layout and reduces pipes, pumps etc. and finally reduces the DEMO plant cost. The final verification of the material properties of the PFCs is pending due to the fact, that irradiation test facility DONES is in the engineering design phase and the constructed is planned to start around 2020 [11]. Adaptions after first irradiation results might change some of the assumptions made here.

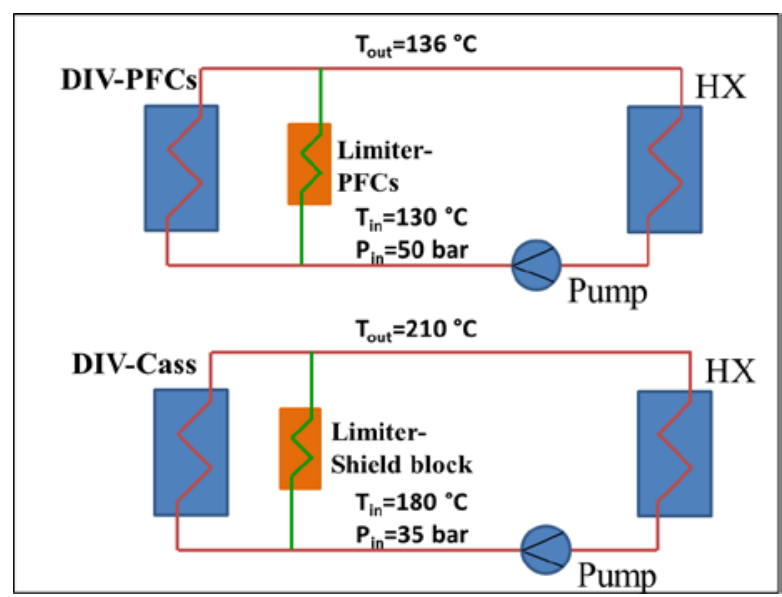

Fig. 5. Limiter cooling in parallel to divertor cooling, it is simplified by applying divertor cooling conditions, courtesy I. Moscato.

\subsection{Electromagnetic (EM) studies}

In the assessment of the electromagnetic loads (EM) due to the fast plasma events the shield block (movable part) was assumed as a box structure with single point electrical connection to the VV. The occurring eddy current loops were determined by means of a finite element model as shown in Fig. 6.

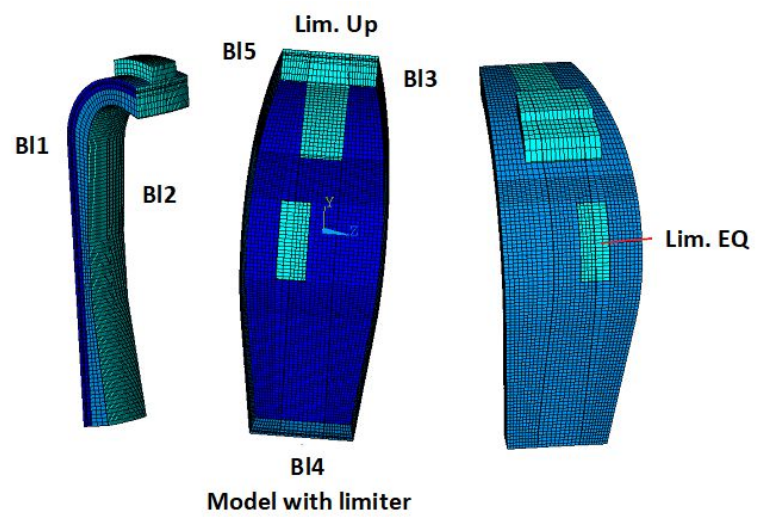

Fig. 6. EM model for simulation of the Maxwell forces. $(\mathrm{Bl} 1=$ left inboard blanket segment, $\mathrm{Bl} 2$ = right inboard blanket segment, Bl3 = left outboard blanket segment, Bl4 = central outboard blanket segment, $\mathrm{Bl5}=$ right outboard blanket segment, Lim. Up = upper limiter, Lim. $\mathrm{EQ}=$ equatorial outboard limiter)

The radial moment was found to be most significant (3.6 MNm), the moments about the vertical and toroidal axes are less severe $(0.5 \mathrm{MNm}$ and $0.1 \mathrm{MNm}$ respectively). Significant net forces on the shield block exist due to gravity ( 6.7 tons) and due to the ferromagnetic steel (Eurofer) in the approximately static magnetic field, see Table 2. The impact of the (changing) poloidal field on the ferromagnetic forces is small. Halo currents are assumed not to occur at the outboard midplane.

Table 2. Ferromagnetic forces on the limiter shield block in different operation phases (negative radial forces pull the shield block towards the plasma).

\begin{tabular}{|c|c|c|c|}
\hline$[\mathrm{MN}]$ & $\begin{array}{c}\text { Dwell } \\
\text { TF coils on, } \\
\text { no plasma, } \\
\text { PF coils off }\end{array}$ & $\begin{array}{c}\text { Operation } \\
\text { TF coils on, } \\
\text { plasma on, } \\
\text { PF coils off }\end{array}$ & $\begin{array}{c}\text { Operation } \\
\text { TF coils on, } \\
\text { plasma on, } \\
\text { PF coils on }\end{array}$ \\
\hline F_rad & -0.39 & -0.43 & -0.52 \\
\hline F_tor & +0.015 & -0.0002 & -0.013 \\
\hline F_vert & -0.004 & -0.0007 & +0.004 \\
\hline
\end{tabular}

The shield block weight could be further reduced, when using materials like boron carbide (B4C)/water or tungsten carbide (WC)/water instead of Eurofer/water.

\subsection{Alignment mechanism}

The question whether an actuated alignment mechanism is needed or a precise assembly would be sufficient was part of a discussion among experts from DEMO and ITER. In ITER formerly a limiter module was proposed, which had 6 degrees of freedom [12], but eventually abandoned. Instead ITER relies on FW panels fixed to shielding blankets that are carefully and individually aligned during assembly [13]. Mechanical adjustment is needed for all individual blanket modules by flexible support cartridges and customized machining on components. From the return of experience (RoX) from ITER and since DEMO is using 'sacrificial' limiters (sacrificial in terms of dedicated elements to allow plasma wall contact) it is proposed to foresee an actuated alignment concept. This allows adjustment of the limiters by means of a reliable actuator system operated from the control room. The alignment mechanism and its components are shown in Fig. 7.

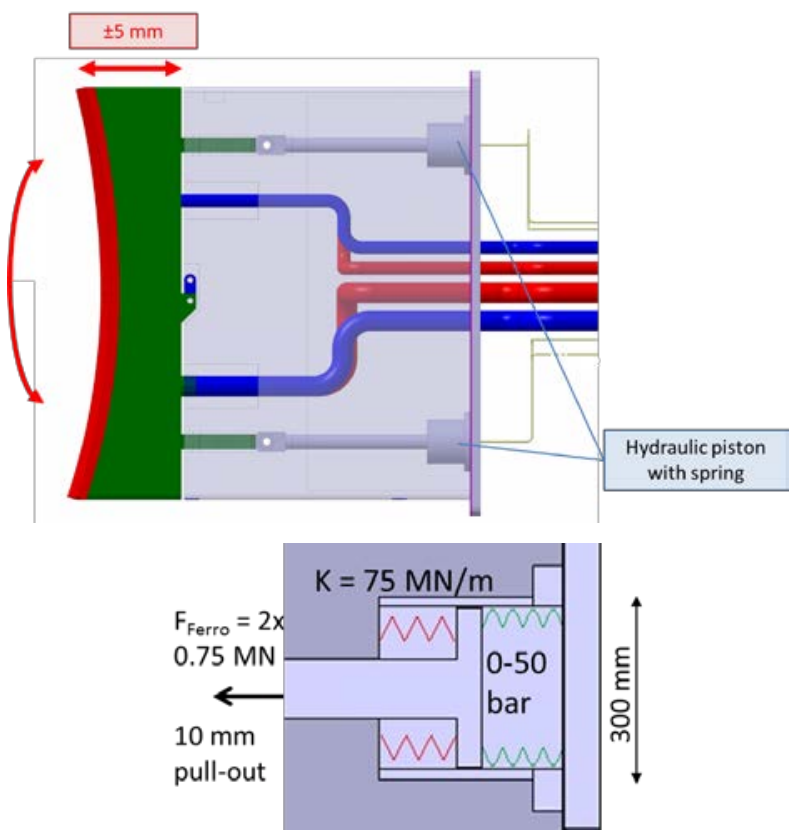

Fig. 7. Limiter alignment mechanism integrated in the limiter (top) and zoomed out (bottom), consists of 2 hydraulic pistons actuated with bellows (green) under variable (controlled) pressure in the range $0-50 \mathrm{bar}(\mathrm{He})$ with springs (red) to react 
on magnetic forces by mainly the toroidal magnetic field. (For interpretation of the references to color in this figure legend, the reader is referred to the web version of this article).

The pneumatic bellow actuator (fed by Helium) with pneumatic pipes for each cylinder penetrates from the permanent part of the port flange into the port and acts on a counter spring. This general design was qualified for the EC launchers (RoX of ITER [14]) and extrapolated in a modified way to DEMO. With a spring, the mechanism has to react first against the static EM force which allows a protrusion of not more than $10 \mathrm{~mm}$ to the FW. Then a pressurized bellow comes into play and allows a fine adjustment of $( \pm 5 \mathrm{~mm})$ by changing the pressure. A backup solution is also foreseen without springs, in which two pneumatic bellows act against each other by applying differential pressure to them. After actuation a brake system radially fixes the alignment rods and reacts with the forces acting on the shield block.

\subsection{Neutronic studies}

Monte Carlo N-Particle (MCNP) code assessments were carried out in order to assess the limiter design against regarding the following limits (and are ongoing):

- Gaps analysis (requirements for doglegs)

- Dpa in materials (PFCs/shield block per full power year (fpy) and for vacuum vessel (VV) (6 fpy = DEMO lifetime)

- Helium production (at pipe re-welding zones)

- Shut-down dose rate assessment (in port interspace, in cryostat, in port cell)

- Nuclear heating in TF and PF coils

No studies were performed on the actuators, since these are well recessed and protected behind the PFCs and shield block from neutrons. Fig. 8 shows the applied limits for the neutronic simulations.

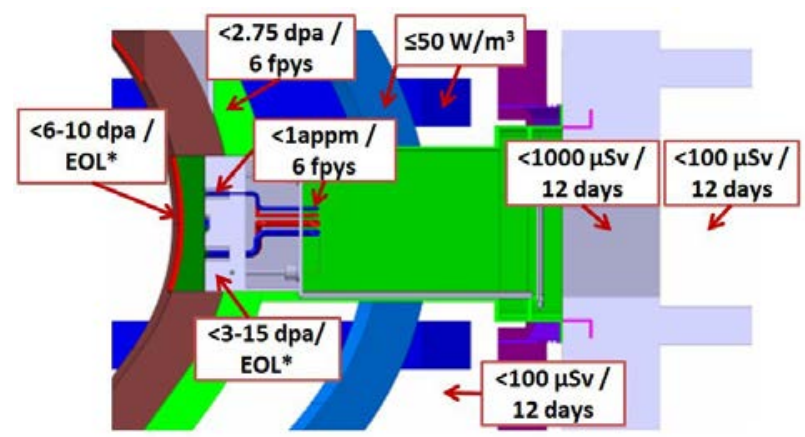

Fig. 8. Neutron shielding requirements for MCNP simulations. *EOL $=$ End Of Life.

The results for the neutron transport analysis as part of the MCNP simulations led to encouraging results and a confirmation that the irradiation damage limit of the $\mathrm{VV}$, as well as the coil heating are within the design limits, see Fig. 9 and Fig. 10.
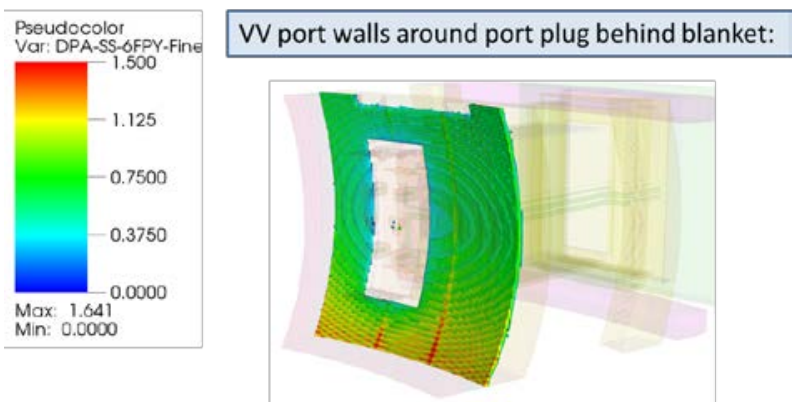

Fig. 9. Results of neutron shielding analysis for dpa in VV. The limit is $<2.75 \mathrm{dpa} / 6 \mathrm{fpy}$, which is well above the achieved results.

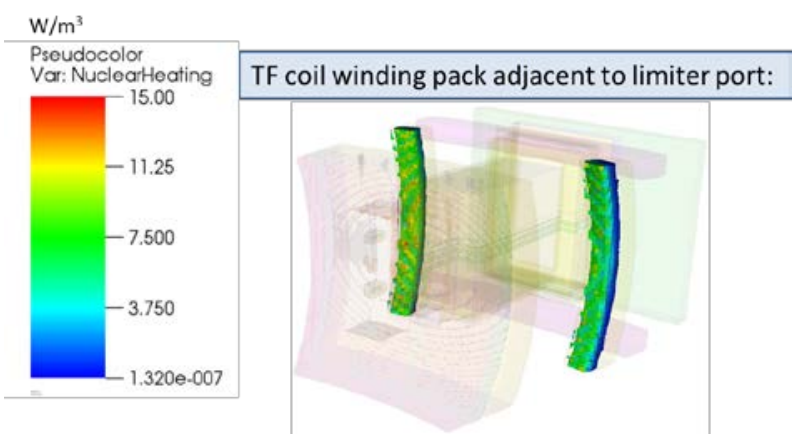

Fig. 10. Results of neutron shielding analysis for TF coil heating. The limit is $<50 \mathrm{~W} / \mathrm{m}^{3}$ for the winding pack of the TF coil, which is well above the achieved results.

The shield block material is Eurofer, the same material as foreseen for the divertor cassette. The aim of the studies to verify an assumed limit of 6-10 dpa per fpy [15] (the assumed limiter lifetime) could be achieved, see Fig. 11.

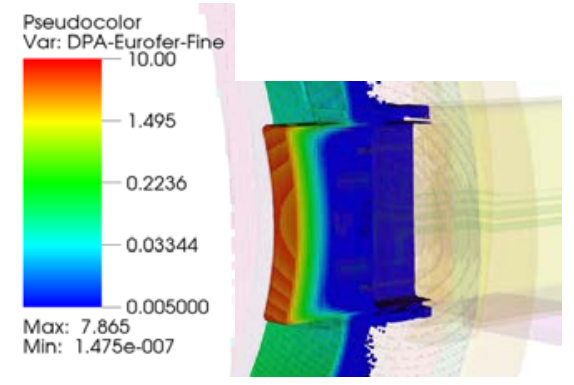

Fig. 11. Dpa / fpy in Eurofer shield block. The front part is exposed up to $10 \mathrm{dpa} / \mathrm{fpy}$. The dpa's in the back part are negligible.

Further neutronic studies were performed on the pipe re-welding zones, see Fig. 12.

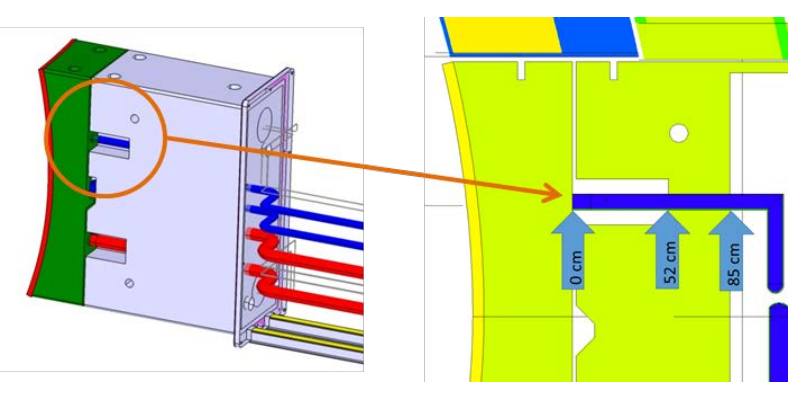




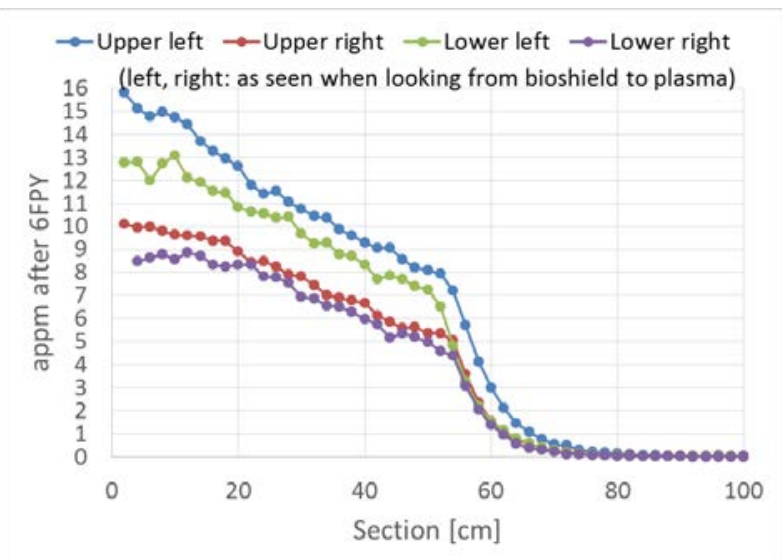

Fig. 12. Helium generation in pipe material at position as indicated in the figure (top), curves for different distances from pipe connection to shield block) after 6 fpys (bottom).

The area in which the pipe disconnection/re-connection takes place is between the shield block and the PFC. The limit condition of 1 appm after 6 fpys was not achieved, see Fig. 12, it was exceeded by a factor of 10 . Additional shielding in form of clips around the cut-outs for the pipe re-welding zones is expected to solve this issue (first results with stainless steel and B4C clips show that the values can be reduced into the right direction).

The results from the neutron transport calculations shown before are promising. Neutronic studies on other equatorial port plugs approximately verify these results [16]. For the SDDR the situation is different. The main information from ITER was to include doglegs or even double-doglegs around the port plug. Since a step-wise approach was envisaged as first step a case without doglegs was assessed. The SDDR limits were exceeded see Fig. 13 and confirmed the need to introduce doglegs. In addition the port plug box design was optimized for improved shielding performance. For the SDDR the lower port was artificially closed as the design was not completely finished. The vertical port showed a nonnegligible cross-talk to the equatorial port (and vice versa).

\section{$\mathrm{X}-\mathrm{Z}$ slice at $\mathrm{Y}=\mathbf{5 0}$, which is through the equatorial port}

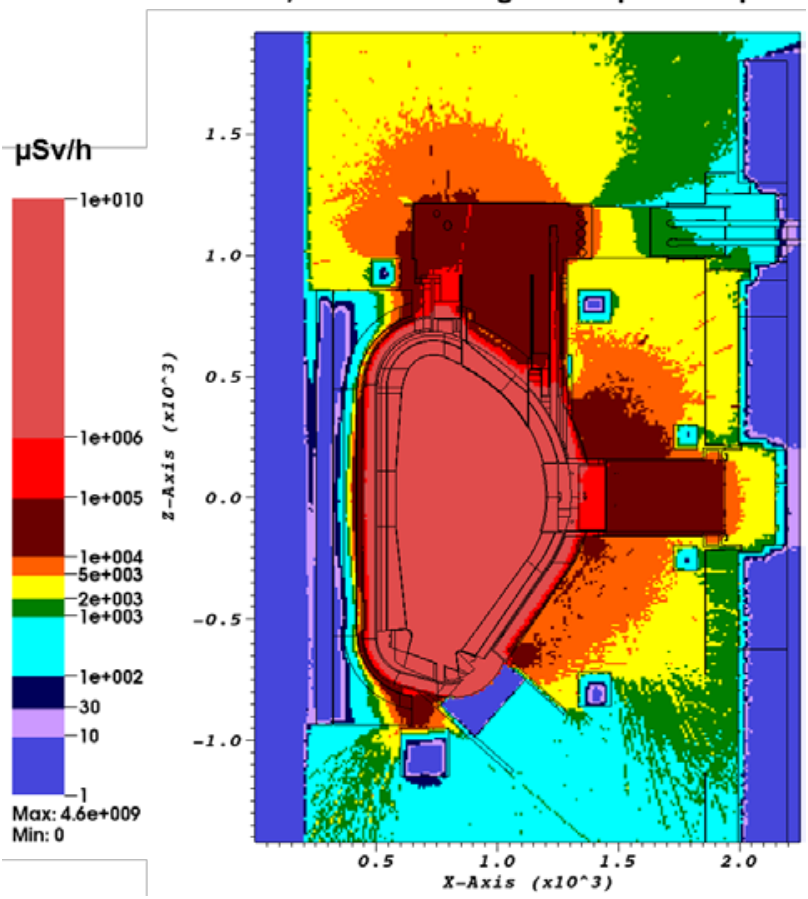

Fig. 13. Gamma radiation results after 12 days of shut down: in port cell about $<30 \mu \mathrm{Sv} / \mathrm{h}$ (limit $10 \mu \mathrm{Sv} / \mathrm{h}$ ) nearly achieved, inside cryostat $2,000-100,000 \mu \mathrm{Sv} / \mathrm{h}$ by far too high (limit $100 \mu \mathrm{Sv} / \mathrm{h}$ ) to allow man access in cryostat area.

\section{Limiter maintenance strategy}

Maintenance considerations have been included in the design from the beginning and regular meetings with the RM work package team were used to implement these. Due to the expected radiation field (Fig. 13) remote solutions will be necessary to protect human operators. The most important design inclusions are summarized below:

- Twist lock handling features added to the back of the port plug to aid in removal from the port along using rail features.

- Further twist lock handling features added to the top of the port plug to allow transportation to the Active Maintenance Facility (AMF) and manipulation within the AMF.

- Remote Maintenance features to allow the unlocking of the shield block/PFC by side access, to simplify the Port Plug maintenance in the AMF without the need for rotation of the Port Plug itself.

- The design includes space reservations for deployment of pipe servicing solutions, whether remote cutting or welding tool or mechanical pipe connector actuators.

\section{Limiter diagnostic system and limiter alignment strategy}

The main diagnostics is to measure PFC cooling water temperature by thermocouples. Also a diagnostics study was successfully performed to allow optionally the infrared (IR) thermography of the limiter PFCs in realtime [17] from neighboring ports. The diagnostics is 
comprised by a position sensor (not studied yet as e.g. strain gauges).

For the assembly and during operation in shut-down phases the procedure for the adjustment of the limiter is based on the installation concept of the ITER first wall. Since in ITER no dedicated limiters are foreseen the ITER first wall needs to be aligned precisely [18]. This is achieved by measuring the toroidal field, measuring the vessel shape by laser tracking, and custom machining and individually adjusting the wall segments as explained in chapter 3.4. For the DEMO limiters a similar procedure is foreseen during assembly. In addition, to relax the alignment precision requirement and to allow for alignment adjustment in operating phase, e.g. after limiter replacement, radial actuators are integrated in the limiter port plug. In order to measure the mutual alignment amongst the ramp-up limiters it is foreseen to operate with a small test plasma, which has much lower plasma current compared to the nominal fusion plasma and could stay for a long time in contact with the limiter PFCs. The temperature rise in the PFCs can be measured either by means of thermocouples in the cooling water or by IR thermography of the PFC surfaces. The actuators will then be used to equalize the power loads on all OMLs.

\section{Summary}

The integrated design of the equatorial port plug limiter was made and EM, neutronic and diagnostic IR thermography studies were conducted. Moderate design improvements are necessary to limit the Helium generation in pipes. The shielding design requires improvements to reduce the SDDR. The basic limiter diagnostic consist of thermocouples and a position sensor, optionally IR thermography was investigated. An alignment strategy was proposed which is based on creating a test-plasma with a low plasma current compared to the nominal fusion plasma current.

The following outstanding design features characterize the OML design:

- Mechanical integrity of the breeding blanket segments is maintained with the integration concept and a toroidal slim limiter design.

- Radial adjustability to align for manufacturing and assembly tolerances, e.g. of the magnetic axis and first wall displacements.

- Flexible pipes (U-bends) and mechanical supports (toroidal keys) allow radial alignment.

- Shared cooling circuits with the divertor at the same pressure / temperature conditions simplifies the plant layout.

- Simplified remote handling procedures by modular and compact design.

- Reduction of nuclear waste by possibility to reuse main limiter sub-components (e.g. the port plug) after necessary regular exchange of PFCs and / or shield block due to end of lifetime (dpa) or accident cases.

\section{Acknowledgments}

This work has been carried out within the framework of the EUROfusion Consortium and has received funding from the Euratom research and training programme 2014-2018 and 2019-2020 under grant agreement No 633053. The views and opinions expressed herein do not necessarily reflect those of the European Commission.

\section{References}

[1] T. Donné et al., J. Instrum. 12, 2017.

[2] G. Federici et al., Fusion Eng. Des. 136 (Part A), (2018), 729-741.

[3] C. Bachmann et al., Key design integration issues addressed in the EU DEMO pre-concept design phase, Fusion Eng. Des. 156, (2020), 111595, (https://doi.org/10.1016/j.fusengdes.2020.111595).

[4] F. Maviglia et al., Impact of Plasma Thermal Transients on the design of the EU DEMO, this conference.

[5] Z. Vizvary, European DEMO First Wall Shaping and Limiters Design and Analysis Status, This Conference.

[6] R. Wenninger, et al., The DEMO wall load challenge, Nucl. Fusion 57, p. 046002, (2017).

[7] M. Mattei, Final Report 2018, EM Investigations of Dynamic Phases in DEMO, EFDA_D_2LFKLN.

[8] T. Franke, et al., Initial port integration concept for EC and NB systems in EU DEMO tokamak, Fusion Eng. Des. 146 (Part B), (2019), 1642-1646, (https://doi.org/10.1016/j.fusengdes.2019.03.007).

[9] G. Mazzone et al, Choice of a low operating temperature for the DEMO EUROFER97 divertor cassette, Fusion Eng. Des. 124, (2017), 655-658.

[10] J.H. You, et al., European DEMO divertor target: operational requirements and material-design interface, Nuclear Mater. Energy 9, (2016), 171-176.

[11] A. Ibarra et al., The IFMIF-DONES project: preliminary engineering design, Nucl. Fusion 58, No. 10, (2018).

[12] V. Rozov, A. Cardella, K. Ioki, Fusion Eng. Des. 73 (2005) 57-82.

[13] R. Raffray et al, The ITER blanket system design challenge, Nucl. Fusion 54, (2014) 033004.

[14] M. A. Henderson et al., The front steering launcher design for the ITER ECRH Upper Port, J. Phys.: Conf. Ser. 25, (2005), 143.

[15] DEMO Plant Description Document (PDD), version 1.5, EFDA_D_2KVWQZ.

[16] A. Cufar et al., Equatorial electron cyclotron port plug neutronic analyses for the EU DEMO, Fusion Eng. Des. 146, (Part A) (2019), 336-340, (https://doi.org/10.1016/j.fusengdes.2018.12.062).

[17] W. Gonzalez et al., Conceptual studies on spectroscopy and radiation diagnostic systems for plasma control on DEMO, Fusion Eng. Des. 146 (Part B), (2019), 22972301, (https://doi.org/10.1016/j.fusengdes.2019.03.176).

[18] B. Macklin et al., Assembly and remote handling of ITER plasma facing components, IEEE Xplore, (2016), (https://ieeexplore.ieee.org/document/7482266) 